\title{
THE SEPARATION PROPERTIES FOR CLOSURES OF TORIC ORBITS
}

\author{
O. V. CHUVASHOVA
}

\begin{abstract}
A subset $X$ of a vector space $V$ is said to have the "Separation Property" if it separates linear forms in the following sense: given a pair $(\alpha, \beta)$ of linearly independent forms on $V$ there is a point $x$ on $X$ such that $\alpha(x)=0$ and $\beta(x) \neq 0$. A more geometric way to express this is the following: every homogeneous hyperplane $H \subseteq V$ is linearly spanned by its intersection with $X$. The separation property was first asked for conjugacy classes in simple Lie algebras.

We give an answer for orbit closures in representation spaces of an algebraic torus. We consider also the strong and the weak separation properties. It turns out that toric orbits well illustrate these concepts.
\end{abstract}

\section{INTRODUCTION}

1. Let $V$ be a vector space over a field $k$ and $G$ be a connected reductive algebraic group. Consider a linear action $G: V$. Properties of the closure of an orbit $\overline{G v} \subset V$ can be sorted into four groups:

(1) "combinatorial" (the number of orbits in $\overline{G v}$, the graph of the orbit adherence relation, ...),

(2) geometrical (the smoothness, the normality, the Cohen-Macaulay property, types of singularities, ...),

(3) topological (the contractibility, the simple connectedness, the computation of homologies and cohomologies, higher homotopy groups, ...),

(4) properties of the embedding $\overline{G v} \subset V$ (the dimension of the linear span, hyperplane sections, a description of the ideal defining the variety, ...).

In this paper we consider the separation properties. In our opinion they belong to the most natural properties of the fourth group.

Definition 1. A subset $X$ of a vector space $V$ has the separation property (briefly (SP)) if for any pair of linearly independent linear functions $\alpha, \beta \in$ $V^{*}$ there exists a point $x \in X$ such that $\alpha(x)=0$ and $\beta(x) \neq 0$.

In other words, the separation property for $X \subset V$ means that $H \cap X \nsubseteq H^{\prime}$ for any pair $H \neq H^{\prime}$ of homogeneous hyperplanes in $V$. Or, equivalently, for any homogeneous hyperplane $H$ the intersection $H \cap X$ linearly spans $H$.

Date: September 23, 2004.

2000 Mathematics Subject Classification. Primary 14C20, 14M25; Secondary 14L30, $14 \mathrm{R} 20$.

Key words and phrases. Toric orbits, hyperplane sections, separation properties, binomial varieties, binary forms. 
For projective spaces we have a similar definition: a subset $Y \subset \mathbb{P}(V)$ has the separation property if for any pair $D \neq D^{\prime}$ of hyperplanes in $\mathbb{P}(V)$ we have $D \cap Y \nsubseteq D^{\prime}$.

Remark 1. Let $\alpha \in V^{*}$ and $H_{\alpha}$ be the corresponding hyperplane. If either $X \cap H_{\alpha}$ is empty or $X \cap H_{\alpha}$ is zero or $X \subseteq H_{\alpha}$, then it is easy to see that $X$ has not (SP).

For the first time the separation property was asked by J. C. Jantzen in connection with the paper of A. Premet [5]:

Question. Let $k$ be an algebraically closed field, $G$ be a simple algebraic group, and $\mathfrak{g}$ be its tangent algebra. Is it true that the minimal nilpotent orbit of the adjoint action in $\mathfrak{g}$ has the separation property?

The answer was obtained in the work [4. It is affirmative for all simple groups except for $S p_{2 n}$.

Example 1. Consider the Lie algebra $\mathfrak{s l}_{2}$. Here the minimal nilpotent orbit consists of the following matrices:

$$
M=\left\{\left(\begin{array}{cc}
a & b \\
c & -a
\end{array}\right): a^{2}+b c=0\right\} .
$$

It is easy to see that $M$ has not the separation property. In fact, $b=0$ implies $a=0$ for a matrix from $M$.

The notions of the "strong" and the "weak" separation properties also were introduced in [4].

Definition 2. A closed affine cone $X \subseteq V$ of dimension $\geq 2$ has the strong separation property (briefly (SSP)) if for any linear subspace $W \subseteq V$ of codimension 2 we have $\operatorname{codim}_{\mathrm{X}} \mathrm{W} \cap \mathrm{X}=2$.

There is a similar definition for a closed projective subvariety. A closed subvariety $Y \subseteq \mathbb{P}(V)$ has the strong separation property if for any linear subspace $L \subseteq \mathbb{P}(V)$ of codimension 2 we have codim $\mathrm{Y} L \cap \mathrm{Y}=2$.

Remark 2. The strong separation property for a closed projective subvariety $Y \subseteq \mathbb{P}(V)$ (closed affine cone $X \subseteq V$ ) implies the separation property.

Proof. Consider the projective case. Suppose that (SP) does not hold. This means that there exist two different hyperplanes $H_{\alpha}$ and $H_{\beta}$ in $\mathbb{P}(V)$ such that $H_{\alpha} \cap Y \subseteq H_{\beta}$. Then $\left(H_{\alpha} \cap H_{\beta}\right) \cap Y=H_{\alpha} \cap Y$ has the codimension $\leq 1$ in $Y$ (see [6, Th. 1.6.4]) and (SSP) does not hold.

In the affine case it is sufficient to notice that (SP) (resp. (SSP)) for a closed affine cone is equivalent to (SP) (resp. (SSP)) for its projectivization.

The next example shows that (SP) does not imply (SSP).

Example 2. Consider the subvariety of degenerate matrices in the space of all $2 \times 2$ matrices:

$$
N=\left\{\left(\begin{array}{ll}
a & b \\
c & d
\end{array}\right): a d-b c=0\right\}
$$


It has not (SP) as it contains the subspace $a=b=0$. By direct calculation it is easy to check that $N$ has (SP). (It also follows from Theorem 3 of this work. Consider the linear action $T=\left(k^{*}\right)^{3}: k^{4},\left(t_{1}, t_{2}, t_{3}\right)\left(x_{1}, x_{2}, x_{3}, x_{4}\right)=$ $\left(t_{1} x_{1}, t_{3} x_{2}, t_{1} t_{2} x_{3}, t_{2} t_{3} x_{4}\right)$. Note that $N$ is isomorphic to $\overline{T(1,1,1,1)}$.)

Definition 3. A subset $X$ of a vector space $V$ has the weak separation property (briefly (WSP)) if for any pair of homogeneous hyperplanes $H \neq H^{\prime}$ we have $H \cap X \neq H^{\prime} \cap X$ (in the set-theoretical sense).

The definition of the weak separation property for a subset of a projective space is similar.

It is obvious that the separation property implies the weak separation property.

Example 3. It is easy to check that the subvariety $M$ of nilpotent matrices in $\mathfrak{s l}_{2}$ has (WSP) and has not (SP). (This also follows from Theorems 3 and 4 of this work. Consider the linear action $T=\left(k^{*}\right)^{2}: k^{3},\left(t_{1}, t_{2}\right)\left(x_{1}, x_{2}, x_{3}\right)=$ $\left(t_{1} t_{2} x_{1}, t_{1}^{2} x_{2}, t_{2}^{2} x_{3}\right)$. Note that $M$ is isomorphic to $\overline{T(1,1,1)}$.)

The following theorems are proved in [4].

Theorem [4, Th. 1]. Let $G$ be a connected semisimple group and $V(\lambda)$ be its Weyl module corresponding to a highest weight $\lambda$ with numerical marks $n_{i}$. Denote by $O_{\min } \subset V(\lambda)$ the orbit of a highest vector. Then:

(1) $\overline{O_{\min }}$ satisfies $(S S P) \Longleftrightarrow \lambda$ is a fundamental weight;

(2) $O_{\min }$ satisfies $(S P) \Longleftrightarrow n_{i} \leq 1$ for any $i$;

(3) $O_{\min }$ satisfies $(W S P) \Longleftrightarrow n_{i} \leq 2$ for any $i$.

Theorem [4] Th. 2]. Let $G: V$ be an irreducible representation of a connected semisimple group $G$. If $O_{\min }$ satisfies (SP), then any $G$-orbit in $V$ satisfies (SP).

Theorem [4, Th. 3]. Suppose that char $k=0$ and $G: V$ is an irreducible representation of a semisimple group $G$. Then a generic $G$-orbit in $V$ satisfies (SP).

2. The aim of this work is to investigate the separation properties for closures of toric orbits in vector and projective spaces over an algebraically closed field. This is a primary generalization of Theorem [4, Th. 1] to the case of reducible representations of reductive groups.

Let $T$ be an algebraic torus, $\Lambda$ be the lattice of characters of $T$, and $V$ be a vector space over an algebraically closed field $k$. Consider a linear action $T: V$, where

$$
t\left(x_{1}, \ldots, x_{n}\right)=\left(\chi_{1}(t) x_{1}, \ldots, \chi_{n}(t) x_{n}\right) .
$$

Let $\Sigma$ be the semigroup in $\Lambda$ generated by the characters $\chi_{1}, \ldots, \chi_{n}$ and $K$ be the cone in $\Lambda \otimes_{\mathbb{Z}} \mathbb{Q}$ generated by $\Sigma$.

Theorem 3. The orbit closure $X=\overline{T(1, \ldots, 1)} \subset V$ satisfies $(S P)$ if and only if the following conditions hold:

(1) the cone $K$ is strictly convex;

(2) $\mathbb{Q}_{+} \chi_{i}$ is an edge of $K$ for any $i$;

(3) $\mathbb{Q}_{+} \chi_{i} \neq \mathbb{Q}_{+} \chi_{j}$ for $i \neq j$.

For a projective action of a torus we have: 
Theorem 3'. The orbit closure $X=\overline{T(1: \ldots: 1)} \subset \mathbb{P}(V)$ satisfies $(S P)$ if and only if the following conditions hold:

(1) the point $\chi_{i}$ is a vertex of the convex hull conv $\left\{\chi_{1}, \ldots, \chi_{n}\right\}$ for any $i$;

(2) $\chi_{i} \neq \chi_{j}$ for $i \neq j$.

The proofs are based on the fact that if the separation property fails on some pair of hyperplanes, then there exist such $T$-invariant hyperplanes. To prove this statement we introduce the notion of the characteristic variety of a subset $X \subset V($ or $X \subset \mathbb{P}(V))$ :

$C h(X)=\left\{(\langle\alpha\rangle,\langle\beta\rangle) \in \mathbb{P}\left(V^{*}\right) \times \mathbb{P}\left(V^{*}\right) \mid \alpha(x)=0 \Longrightarrow \beta(x)=0 \quad \forall x \in X\right\}$.

After that we prove:

Theorem 2. Suppose that an affine subvariety $X \subset V$ is irreducible, is not contained in a homogeneous hyperplane, meets any homogeneous hyperplane and $\operatorname{dim} X>1$. Then $C h(X)$ is closed in $\mathbb{P}\left(V^{*}\right) \times \mathbb{P}\left(V^{*}\right)$.

Finally we apply the fact that an algebraic torus acting on a projective variety has a fixed point. More precisely:

Proposition 3. If $X$ does not satisfy $(S P)$ and $C h(X)$ is closed, then there exists a pair $(\langle\alpha\rangle,\langle\beta\rangle) \in C h(X)$ such that $\alpha, \beta$ are $T$-semiinvariant and linearly independent.

Proposition 3 allows to simplify the proof of the criterion of the separation property for $S L_{2}$-orbits of binary forms obtained in the thesis of K. Baur.

Theorem [1, Th. 3.4]. Let $f \in k[x, y]_{n}$. Then the orbit $O_{f}=S L_{2} f$ satisfies $(S P)$ if and only if $f$ has a linear factor of multiplicity one.

For the weak separation property we obtain the following theorems.

Theorem 4. The orbit closure $X=\overline{T(1, \ldots, 1)} \subset V$ satisfies (WSP) if and only if the following conditions hold:

(1) the cone $K$ is strictly convex;

(2) there are no more then one characters $\chi_{i}$ in the interior of any face of $K$ (in particular, $\mathbb{Q}_{+} \chi_{i} \neq \mathbb{Q}_{+} \chi_{j}$ for $i \neq j$ ).

Theorem 4'. The orbit closure $X=\overline{T(1: \ldots: 1)} \subset \mathbb{P}(V)$ satisfies (WSP) if and only if there are no more then one characters $\chi_{i}$ in the interior of any face of the convex hull conv $\left\{\chi_{1}, \ldots, \chi_{n}\right\}$ (in particular, $\chi_{i} \neq \chi_{j}$ for $i \neq j$ ).

Finally we consider the strong separation property.

Theorem 5. Suppose that the orbit closure $X=\overline{T(1, \ldots, 1)} \subset V$ is a cone; then $X$ satisfies (SSP) if and only if $X=V$ (i.e., the weights $\chi_{1}, \ldots, \chi_{n}$ are linearly independent).

Theorem 5'. The orbit closure $X=\overline{T(1: \ldots: 1)} \subset \mathbb{P}(V)$ satisfies $(S S P)$ if and only if $X=\mathbb{P}(V)$ (i.e., the weights $\chi_{1}, \ldots, \chi_{n}$ are affinely independent).

I am grateful to my adviser Ivan V. Arzhantsev for the subject of this work, the permanent support, and numerous remarks and ideas. I also thank Dmitri A. Timashev for important comments and detection of a gap in the preliminary version of the text. 


\section{HyPERSURFACES}

Proposition 1. Let $S \subset V$ be a hypersurface. Then

(1) $S$ does not satisfy $(S P) \Longleftrightarrow S$ can be defined by an equation $x_{2}^{n}+$ $x_{1} F=0$ in some coordinate system for some $n \in \mathbb{N}$ and $F \in k[V]$;

(2) $S$ does not satisfy (WSP) $\Longleftrightarrow S$ can be defined by an equation $x_{1}^{n}+x_{2}^{m}+x_{1} x_{2} F=0$ in some coordinate system for some $n, m \in \mathbb{N}$ and $F \in k[V]$

(3) a homogeneous hypersurface $S$ does not satisfy $(S S P) \Longleftrightarrow S$ can be defined by an equation $x_{1} F_{1}+x_{2} F_{2}=0$ in some coordinate system for some homogeneous polynomials $F_{1}, F_{2} \in k[V]$ of the same degree.

Proof. Let $S$ be defined by an equation $P=0$. We may assume that $P$ has no multiple factors. Since the field $k$ is algebraically closed, it follows that $P$ is defined uniquely up to a constant.

(1) If $P$ has the form $x_{2}^{n}+x_{1} F$ in some coordinate system, then $S \cap H_{x_{1}} \subseteq H_{x_{2}}$ and $S$ has not (SP).

Conversely, if $S$ has not (SP), then we can choose a coordinate system such that $S \cap H_{x_{1}} \subseteq H_{x_{2}}$. This means that $P\left(0, x_{2}, \ldots\right)=0$ implies $x_{2}=0$. By Hilbert's nullstellentsatz, we have $x_{2}^{l}=P\left(0, x_{2}, \ldots\right) f\left(x_{2}, \ldots\right)$. This implies $P\left(0, x_{2}, \ldots\right)=c x_{2}^{n}$ for some $n \in \mathbb{N}, n \leq l$, and $c \in k^{*}$. Then $P=c x_{2}^{n}+x_{1} F$. (We may assume that $c=1$.)

(2) If $P$ has the form $x_{1}^{n}+x_{2}^{m}+x_{1} x_{2} F$ in some coordinate system, then $S \cap H_{x_{2}}=S \cap H_{x_{1}}$ and $S$ has not (WSP).

Conversely, if $S$ has not (WSP), then we can choose a coordinate system such that $S \cap H_{x_{2}}=S \cap H_{x_{1}}$. Statement (1) implies that in this coordinate system $P=x_{1}^{n}+x_{2} F_{1}=x_{2}^{m}+x_{1} F_{2}$ (up to the proportionality of the basis vectors). This implies $P=x_{1}^{n}+x_{2}^{m}+x_{1} x_{2} F$.

(3) If $P$ has the form $x_{1} F_{1}+x_{2} F_{2}$ in some coordinate system, then the subspace in $V$ defined by the equations $x_{1}=0, x_{2}=0$ has the codimension one in $S$ and $S$ has not (SSP).

Conversely, if $S$ has not (SSP), then we can choose a coordinate system such that the subspace in $V$ defined by the equations $x_{1}=0, x_{2}=0$ has the codimension $\leq 1$ in $S$ (this implies that this subvariety is contained in $S$ ). The ideal generated by the polynomials $x_{1}, x_{2}$ is radical, so, by Hilbert's nullstellentsatz, $P=x_{1} F_{1}+x_{2} F_{2}$.

Denote by $I(X)$ the ideal of a closed affine subvariety $X \subseteq V$.

Proposition 2. Let $X \subseteq V$ be a closed affine subvariety. Then

(1) $X$ does not satisfy (SP) if and only if $X$ is contained in a hyperpsurface $S$ such that $S$ does not satisfy $(S P)$;

(2) $X$ does not satisfy (WSP) if and only if $X$ is contained in a hypersurface $S$ such that $S$ does not satisfy (WSP).

Proof. Suppose that $X$ has (SP) (resp. (WSP)). It is evident that any subset $Z$ in $V$ such that $X \subseteq Z$ has (SP) (resp. (WSP)).

Now we shall prove the converse implication. Suppose that $X$ has not (SP). Then there exist linearly independent $\alpha, \beta \in V^{*}$ such that $H_{\alpha} \cap X \subseteq H_{\beta}$. By Hilbert's nullstellentsatz, this holds if and only if 
$\beta^{n}=f+g \alpha$ for some $n \in \mathbb{N}, g \in k[V], f \in I(X)$. Let $S$ be the zero set of $f$. Then $X \subseteq S$ and, by Proposition $1, S$ has not (SP).

Suppose that $X$ has not (WSP). Then there exist linearly independent $\alpha, \beta \in V^{*}$ such that $H_{\alpha} \cap X=H_{\beta} \cap X$. Let $F$ be the hypersurface in $V$ defined by the equation $\alpha(x) \beta(x)=0$. The equality $H_{\alpha} \cap X=H_{\beta} \cap X$ is equivalent to two equalities: $H_{\alpha} \cap X=F \cap X$ and $H_{\beta} \cap X=F \cap X$. They hold if and only if $\operatorname{rad}(I(X), \alpha)=\operatorname{rad}(I(X), \alpha \beta)$ and $\operatorname{rad}(I(X), \beta)=$ $\operatorname{rad}(I(X), \alpha \beta)$ (here $(I(X), f)$ is the ideal generated by $I(X)$ and $f)$. We have

$$
\begin{aligned}
\alpha^{k} & =f_{1}+g_{1} \alpha \beta, \\
\beta^{l} & =f_{2}+g_{2} \alpha \beta
\end{aligned}
$$

for some $k, l \in \mathbb{N}, f_{1}, f_{2} \in I(X), g_{1}, g_{2} \in k[V]$. Summing these equations, we get $f_{1}+f_{2}=\alpha^{k}+\beta^{l}-\alpha \beta\left(g_{1}+g_{2}\right)$. Let $S$ be the zero set of the polynomial $f_{1}+f_{2}$. Then $S$ contains $X$ and, by the previous proposition, has not (WSP).

Remark 3. Let us remark that the analogous statement does not hold for (SSP). In fact, let $X$ be a closed affine cone of dimension $\geq 2$ and $f \in I(X)$ be a homogeneous polynomial. Then the homogeneous hypersurface defined by the equation $x_{1} f(x)=0$ contains $X$ and has not (SSP). Moreover, we shall give an example of a closed affine cone having (SSP) and containing in an irreducible hypersurface such that this hypersurface has not (SSP).

Example 4. Let $X \subset V$ be a closed irreducible affine cone of codimension $\geq 2$ having (SSP). There exist homogeneous coprime $f, p \in I(X)$. Consider the subspace $W=V \oplus k^{2}$ ( $x, y$ are coordinates in the last two items). Denote by $S$ the hypersurface in $W$ defined by the equation $f x^{m}+p y=0$ (where $\operatorname{deg} p=\operatorname{deg} f+m-1$ ) and by $Y$ the closed affine cone $X \oplus k^{2} \subset W$. Then $S$ has not (SSP) and is irreducible. Note that $Y \subset S$.

We shall prove that $Y$ has (SSP). It is sufficient to prove that $X \subset V$ has (SSP) implies $X^{\prime}=X \oplus k \subset V^{\prime}=V \oplus k$ has (SSP). Assume the converse. Let (SSP) fail for $X^{\prime}$ on the subspace $U^{\prime} \subset V^{\prime}$ defined by equations $\alpha^{\prime}=\alpha+a z=0, \beta^{\prime}=\beta+b z=0$, where $z$ is a coordinate in the second item of $V \oplus k$.

The first case. Let $\alpha$ and $\beta$ be not proportional. Here

$\operatorname{dim}\left(X \cap H_{\alpha} \cap H_{\beta}\right)=\operatorname{dim}\left(X^{\prime} \cap H_{\alpha^{\prime}} \cap H_{\beta^{\prime}} \cap H_{z}\right) \geq \operatorname{dim} X^{\prime}-2=\operatorname{dim} X-1$

and we obtain a contradiction with the fact that $X$ has (SSP).

The second case. If $\alpha=c \beta$, then $U^{\prime}$ can be defined by the equations $\alpha=0, z=0$. As $\left(X^{\prime} \cap H_{z}\right) \cap H_{\alpha}=X \cap H_{\alpha}$ has the codimension 2 in $X^{\prime}$, we have a contradiction.

Proposition 2'. Let $Y \subseteq \mathbb{P}(V)$ be a closed projective subvariety. Then

(1) $Y$ does not satisfy (SP) if and only if $Y$ is contained in a hypersurface $R$ such that it does not satisfy (SP);

(2) $Y$ does not satisfy (WSP) if and only if $Y$ is contained in a hypersurface $R$ such that it does not satisfy (WSP).

Proof. Consider the vector space $V$ and the closed affine cone $X \subseteq V$ over $Y$. The cone $X$ has (SP) (resp. (WSP)) if and only if $Y$ has (SP) (resp. $(\mathrm{WSP}))$. 
(1) If $Y \subseteq R$ and $R$ has not (SP), then $X \subseteq S$, where $S$ is the affine cone over $R$ in $V$ and $S$ has not (SP). This implies that $X$ has not (SP).

Conversely, if $X$ has not (SP), then, by Proposition 1, there exists a hypersurface $S \subset V$ such that $X \subseteq S$ and $S$ has not (SP). Let $S$ be defined by the equation $f=0$, where $f \in I(X)$. By Proposition $1, f$ has the form $x_{2}^{n}+x_{1} F$ in some coordinate system. As the ideal $I$ is homogeneous, the homogeneous component of degree $n$ of this equation belongs to $I(X)$. This component has the form $x_{2}^{n}+x_{1} F^{\prime}$. Hence the corresponding projective hypersurface contains $Y$ and has not (SP).

(2) If $Y \subseteq R$ and $R$ has not (WSP), then $X \subseteq S$, where $S$ is the affine cone over $R$ in $V$ and $S$ has not (WSP). This implies that $X$ has not (WSP).

If $X$ has not (WSP), then, by Proposition 1, there exists a hypersurface $S \subset V$ such that $X \subseteq S$ and $S$ has not (WSP). Let $S$ be defined by $f=0$, where $f \in I(X)$. By Proposition $1, f$ has the form $x_{1}^{n}+x_{2}^{m}+x_{1} x_{2} F$ in some coordinate system. As the ideal $I(X)$ is homogeneous, the homogeneous components of degrees $n$ and $m$ of this equation belong to $I(X)$. If $n=m$, then this component has the form $x_{1}^{n}+x_{2}^{n}+x_{1} x_{2} F^{\prime}$ and corresponding projective hypersurface contains $Y$ and has not (WSP). If $n \neq m$, then these components have the forms $x_{1}^{n}+x_{1} x_{2} F_{1}$ and $x_{2}^{m}+x_{1} x_{2} F_{2}$. Consider the polynomial $\left(x_{1}^{n}+x_{1} x_{2} F_{1}\right)^{m}+\left(x_{2}^{m}+x_{1} x_{2} F_{2}\right)^{n}$. This homogeneous polynomial of degree $m n$ belongs to $I(X)$ and the projective hypersurface defined by it has not (WSP).

It was proved in 4 that the strong separation property is a property of open type in the following sense. Recall that a family of $d$-dimensional subvarieties in $\mathbb{P}(V)$ is a closed subvariety $F \subset B \times \mathbb{P}(V)$, where $B$ is an algebraic variety such that the projection $p r_{B}$ induces the surjective morphism $p: F \rightarrow B$ and any fiber of this morphism has the dimension $d$.

Proposition [4 Prop. 6]. Let $p: F \rightarrow B$ be a family of d-dimensional closed subvarieties in $\mathbb{P}(V)$. Then the subset $\left\{b \in B \mid p^{-1}(b)\right.$ satisfies $\left.(S S P)\right\}$ is open in $B$.

The following example shows that the weak separation property and the separation property are not properties of open type.

Example 5. Consider the closed family of hypersurfaces $F \subset \mathbb{P}(V) \times k$ defined by the equation $b\left(x_{1}^{m}+x_{2}^{m}\right)+x_{1} x_{2} P=0$, where $b \in k$ and the hypersurface $R \subset \mathbb{P}(V)$ defined by the equation $P=0$ has $(\mathrm{SP})$ ( $\operatorname{deg} P=$ $m-2)$. Then, by Proposition 1 , the set $p^{-1}(b)$ has not (WSP) if $b \neq 0$. The set $p^{-1}(0)$ has (SP), as it contains the subset $R$ having (SP).

The author does not know an example of such family of subvarieties with an irreducible fiber over any point $b \in B$.

\section{Characteristic varieties}

Let $X$ be a subset in a vector space $V$.

Definition 4. The characteristic variety $C h(X)$ of a subset $X$ is the subset in $\mathbb{P}\left(V^{*}\right) \times \mathbb{P}\left(V^{*}\right)$ consisting of the following pairs:

$$
C h(X)=\left\{(\langle\alpha\rangle,\langle\beta\rangle) \in \mathbb{P}\left(V^{*}\right) \times \mathbb{P}\left(V^{*}\right) \mid \alpha(x)=0 \Longrightarrow \beta(x)=0 \quad \forall x \in X\right\} .
$$


Definition 5. The weak characteristic variety $C h w(X)$ of a subset $X$ is the subset in $\mathbb{P}\left(V^{*}\right) \times \mathbb{P}\left(V^{*}\right)$ consisting of the following pairs:

$$
\begin{gathered}
C h w(X)=\left\{(<\alpha>,<\beta>) \in \mathbb{P}\left(V^{*}\right) \times \mathbb{P}\left(V^{*}\right) \mid \alpha(x)=0 \Longleftrightarrow\right. \\
\beta(x)=0 \quad \forall x \in X\} .
\end{gathered}
$$

Remark 4. (1) If $\phi: \mathbb{P}\left(V^{*}\right) \times \mathbb{P}\left(V^{*}\right) \rightarrow \mathbb{P}\left(V^{*}\right) \times \mathbb{P}\left(V^{*}\right)$ is the symmetry with respect to the diagonal, i.e., $\phi((\langle\alpha\rangle,\langle\beta\rangle))=(\langle\beta\rangle,\langle\alpha\rangle)$, then $C h w(X)=$ $C h(X) \cap \phi(C h(X))$;

(2) the diagonal $D=(\langle\alpha\rangle,\langle\alpha\rangle) \subseteq C h w(X) \subseteq C h(X)$;

(3) $X$ satisfies $(\mathrm{SP}) \Longleftrightarrow C h(X)=D$;

$X$ satisfies $(\mathrm{WSP}) \Longleftrightarrow C h w(X)=D$;

We shall need the following theorem.

Theorem 1. 3, Th. 4.5] Let $\phi: X \rightarrow Y$ be a dominant morphism of irreducible varieties and $r=\operatorname{dim} X-\operatorname{dim} Y$. Suppose that for any closed irreducible subvariety $W \subseteq Y$ any irreducible component of $\phi^{-1}(W)$ has the dimension $\operatorname{dim} W+r$. Then $\phi$ is an open morphism.

Theorem 2. Suppose that an algebraic subvariety $X \subset V$ is irreducible, is not contained in a homogeneous hyperplane, meets any homogeneous hyperplane and $\operatorname{dim} X>1$. Then $C h(X)$ and $C h w(X)$ are closed.

Remark 5. If the conditions of the theorem hold for any irreducible component $X_{i}$ of a subvariety $X \subset V$, then $C h(X)=\cap C h\left(X_{i}\right)$ is closed.

Proof. Consider the closed subvariety

$$
M=\left\{(\langle\alpha\rangle,\langle\beta\rangle, x) \in \mathbb{P}\left(V^{*}\right) \times \mathbb{P}\left(V^{*}\right) \times X \mid \alpha(x)=0\right\} .
$$

Let $\phi: M \rightarrow \mathbb{P}\left(V^{*}\right) \times \mathbb{P}\left(V^{*}\right)$ be the projection along the third component. Consider the open subset $L=\{(\langle\alpha\rangle,\langle\beta\rangle, x) \in M: \beta(x) \neq 0\}$ in $M$. Note that $C h(X)=\mathbb{P}\left(V^{*}\right) \times \mathbb{P}\left(V^{*}\right) \backslash \phi(L)$. Thus we need to prove that $\phi(L)$ is open. We shall show that $\phi$ is an open morphism applying Theorem 1.

(1) The variety $M$ is irreducible. We prove this in Lemma 1 below.

(2) The morphism $\phi$ is surjective. Indeed, $\phi(M)=\{(\langle\alpha\rangle,\langle\beta\rangle) \mid \exists x \in X$ : $\alpha(x)=0\}$. But $X$ meets any homogeneous hyperplane.

(3) Let $W \subseteq \mathbb{P}\left(V^{*}\right) \times \mathbb{P}\left(V^{*}\right)$ be closed and irreducible. We have $\phi^{-1}(W)=$ $(W \times X) \cap R$, where $R \subset \mathbb{P}\left(V^{*}\right) \times \mathbb{P}\left(V^{*}\right) \times V$ is defined by the equation $\alpha(x)=$ 0 . Therefore $\phi^{-1}(W)$ is a hypersurface in the irreducible variety $W \times X$ and any irreducible component of $\phi^{-1}(W)$ has the dimension $\operatorname{dim} X+\operatorname{dim} W-1$ $\left(\phi^{-1}(W)\right.$ is not empty as $X$ meets any hyperplane and $\phi^{-1}(W)$ does not coincide with $W \times X$ as $X$ is not contained in a hyperplane).

Thus the morphism $\phi$ is open. This proves Theorem 2 .

Lemma 1. Under the conditions of Theorem 2 the variety $M$ is irreducible.

Proof. It is sufficient to prove that the variety $M^{\prime} \subseteq V^{*} \times X, M^{\prime}=\{(\alpha, x)$ : $\alpha(x)=0\}$, is irreducible.

(1) Consider the variety $L \subseteq V^{*} \times V, L=\{(\alpha, v): \alpha(v)=0\}$, and the projection $\psi: L \rightarrow V$. The morphism $\psi^{\prime}: L \backslash \psi^{-1}(0) \rightarrow V \backslash(0)$ is a vector bundle. Indeed, fix a basis in $V$ and the dual basis in $V^{*}$. Let 
$U_{i}=\left\{v \in V: x_{i} \neq 0\right\}$. Then $\psi^{\prime-1}\left(U_{i}\right) \cong W \times U_{i}$, where $W$ is a vector space of dimension $n-1$ and the isomorphism is defined by the following formula:

$$
\begin{gathered}
\left.\left(a_{1}, \ldots, a_{i-1}, a_{i+1}, \ldots, a_{n}\right),\left(x_{1}, \ldots, x_{n}\right)\right) \rightarrow \\
\left(\left(a_{1}, \ldots, a_{i}=\frac{-1}{x_{i}} \sum_{j \neq i} a_{j} x_{j}, \ldots, a_{n}\right),\left(x_{1}, \ldots, x_{n}\right)\right) .
\end{gathered}
$$

(2) Let $Z=M^{\prime} \backslash \psi^{-1}(0)=\psi^{\prime-1}(X \backslash\{0\})$. The map $\psi^{\prime \prime}: Z \rightarrow X \backslash\{0\}$ is also a vector bundle. The variety $X$ is irreducible, consequently, $X \backslash\{0\}$ is irreducible and $Z$ is irreducible.

(3) We have $M^{\prime}=Z \sqcup Y$, where $Y$ is isomorphic to $V^{*}$ and closed, $Z$ is irreducible. Let $M^{\prime}=\cup M_{i}$, where $M_{i}$ are the irreducible components of $M^{\prime}$. It can be assumed that $\bar{Z} \subseteq M_{1}, Y \subseteq M_{2}$. This yields that there are no more then two irreducible components. If $Y \neq M_{2}$, then $M_{2}=$ $Y \cup\left(M_{2} \cap \bar{Z}\right)$ and we have a contradiction with the irreducibility of $M_{2}$. Hence $Y$ is an irreducible component of $M^{\prime}$. Further, $M^{\prime}$ is a hypersurface in the irreducible variety $X \times V^{*}$ and $\operatorname{dim} M_{i}=\operatorname{dim} M^{\prime}$. At the same time $\operatorname{dim} M^{\prime}=n+\operatorname{dim} X-1>n$ and $\operatorname{dim} Y=n$. So we have a contradiction. This means that $M^{\prime}$ is irreducible.

Now we give some examples when the conditions of Theorem 2 do not hold and $C h(X)$ is not closed.

Example 6. (1) $X$ is a line $\langle v\rangle \subset k^{n}, n \geq 2$ (here $C h(X)$ contains the open subset $\{(\langle\alpha\rangle,\langle\beta\rangle) \mid \alpha(v) \neq 0\}$ of $\left.\mathbf{P}\left(V^{*}\right) \times \mathbf{P}\left(V^{*}\right)\right)$;

(2) $X$ is the subvariety in $k^{3}$ defined by the equations $x_{1}=1$ and $x_{2}=x_{3}$ (indeed, $C h(X)$ contains the subset $\left\{\left(\left\langle\alpha=a_{1} x_{1}+a_{2} x_{2}+a_{3} x_{3}\right\rangle,\langle\beta\rangle\right) \mid a_{1} \neq\right.$ $\left.0, a_{2}+a_{3}=0\right\}$, the pair $\left(\left\langle x_{2}-x_{3}\right\rangle,\left\langle x_{1}\right\rangle\right)$ is contained in its closure and is not contained in $C h(X))$;

(3) $X \subset V=k^{2}$ is defined by the equation $x_{1} x_{2}=0$ (here $C h(X)$ contains the open subset $\left\{\left(\left\langle\alpha=a_{1} x_{1}+a_{2} x_{2}\right\rangle,\langle\beta\rangle\right) \mid a_{1} \neq 0, a_{2} \neq 0\right\}$ of $\left.\mathbf{P}\left(V^{*}\right) \times \mathbf{P}\left(V^{*}\right)\right)$.

Theorem 2 implies the similar theorem for a subset in a projective space (the definition of the characteristic variety in the projective case is analogous).

Theorem 2'. Suppose that an algebraic subvariety $Y \subset \mathbb{P}(V)$ is irreducible and is not contained in a hyperplane. Then $C h(Y)$ and $C h w(Y)$ are closed.

Proof. Let $X \subset V$ be the cone corresponding to $Y$. Note that $C h(X)=$ $C h(Y)$. Applying Theorem 2 to $X$, we conclude that $C h(X)$ is closed.

\section{The CASE OF A T-INVARIANT SUbVARIETY}

Let $T$ be an algebraic torus linearly acting on a vector space $V$ and $X$ be a $T$-invariant subset in $V$. Then $C h(X)$ is a $T$-invariant subset in $\mathbb{P}\left(V^{*}\right) \times \mathbb{P}\left(V^{*}\right)$.

Proposition 3. If $X$ has not the separation property and $C h(X)$ is closed, then there exists a pair $(\langle\alpha\rangle,\langle\beta\rangle) \in C h(X)$ such that $\alpha, \beta$ are $T$ semiinvariant and linearly independent. 
Proof. Fix a $T$-semiinvariant basis $\left\{x_{1}, \ldots, x_{n}\right\}$ in $V^{*}$. Let $\lambda_{i}$ be the weight of $x_{i}$.

There exists a pair $(\langle\alpha\rangle,\langle\beta\rangle) \in C h(X)$ such that $\langle\alpha\rangle \neq\langle\beta\rangle$. Consider the action $T: \overline{T(\langle\alpha\rangle,\langle\beta\rangle)}$. For an action of a torus on a projective variety there exists a fixed point. Denote it by $\left(\left\langle\alpha^{\prime}\right\rangle,\left\langle\beta^{\prime}\right\rangle\right)$. If $\left\langle\alpha^{\prime}\right\rangle \neq\left\langle\beta^{\prime}\right\rangle$, then there is nothing to prove. Now let $\left\langle\alpha^{\prime}\right\rangle=\left\langle\beta^{\prime}\right\rangle$.

There exists a one-parameter subgroup $\gamma: k^{*} \rightarrow T$ such that

$$
\lim _{s \rightarrow 0} \gamma(s)(\langle\alpha\rangle,\langle\beta\rangle)=\left(\left\langle\alpha^{\prime}\right\rangle,\left\langle\alpha^{\prime}\right\rangle\right)
$$

(see [2, Sec. 2.3]).

Let $\left(\alpha_{1}, \ldots, \alpha_{n}\right)$ be the coordinates of $\alpha$, and $\left(\beta_{1}, \ldots, \beta_{n}\right)$ be the coordinates of $\beta$. We may assume that $\alpha^{\prime}=\left(\alpha_{1}^{\prime}, \ldots \alpha_{n}^{\prime}\right)$, where $\alpha_{i}^{\prime}=\alpha_{i}$ if $\left\langle\gamma, \lambda_{i}\right\rangle=\min _{j}: \alpha_{j} \neq 0\left\langle\gamma, \lambda_{j}\right\rangle$, and $\alpha_{i}^{\prime}=0$ in another case, and the formula for $\beta^{\prime}$ is analogous (here $\langle\cdot, \cdot\rangle$ is the natural pairing between the lattice of one-parameter subgroups and the lattice of characters ). Let $\beta^{\prime}=c \alpha^{\prime}$.

We have $(\langle\alpha\rangle,\langle\beta-c \alpha\rangle) \in C h(X)$ and

$$
\lim _{s \rightarrow 0} \gamma(s)(\langle\alpha\rangle,\langle\beta-c \alpha\rangle)=\left(\left\langle\alpha^{\prime}\right\rangle,\left\langle\beta^{\prime \prime}\right\rangle\right) \in C h(X) .
$$

Note that the supports of the vectors $\alpha^{\prime}, \beta^{\prime \prime}$ do not intersect. (Here the support of a vector $v$ is the set of basis vectors along which $v$ has nonzero coordinates; the support of $\langle v\rangle$ is the support of $v$.) Thus we obtain $\overline{T\left(\left\langle\alpha^{\prime}\right\rangle,\left\langle\beta^{\prime \prime}\right\rangle\right)} \cap D=\emptyset$ and the desired point is a $T$-fixed point in $\overline{T\left(\left\langle\alpha^{\prime}\right\rangle,\left\langle\beta^{\prime \prime}\right\rangle\right)}$.

Corollary 1. Let $T: V$ and all $\lambda_{i}$ be different. Let $\left\{\epsilon_{i}\right\}$ be a $T$-semiinvariant basis in $V,\left\{x_{i}\right\}$ be the dual basis in $V^{*}$, and $X \subset V$ be a closed irreducible affine T-invariant subvariety. Then $X$ does not satisfy (SP) $\Longleftrightarrow X$ is contained in a hypersurface of the form $x_{i}^{n}+x_{j} F=0$ for some $i \neq j$, $F \in k[V]$ and $n \in \mathbb{N}$.

Proof. We have to prove that the separation property fails on a pair of $T$ semiinvariant linear functions.

(1) If $X$ is contained in a homogeneous hyperplane, then $X$ is contained in a $T$-invariant homogeneous hyperplane and (SP) fails on a $T$-semiinvariant pair.

(2) If $X$ does not meet a homogeneous hyperplane, then there exist $f, g \in k[X]$, such that $f g=1$. If the functions $f$ and $g$ are not $T$ semiinvariant, then we consider their weight decompositions. Since $k[X]$ has no zero divisors, it follows that after the multiplication in the left side of the equality we obtain the sum of weight functions with different weights. There exists a one-parameter subgroup having different pairing with all weights from the weight decompositions of $f$ and $g$. This one-parameter subgroup defines the order on weight functions. The products of the highest and the lowest terms can not be cancelled, so we have a contradiction. Thus $X$ does not meet a $T$-invariant homogeneous hyperplane.

(3) If $\operatorname{dim} X=1$, then either $X$ is a curve of $T$-fixed points or $X$ is a closure of an orbit of a one-dimensional torus. In the first case $X$ is contained in the subspace defined by the equations $x_{i}=0$, where $\lambda_{i} \neq 0$. 
In the second case (SP) fails on any pair of coordinate functions $x_{i}, x_{j}$ such that either $\lambda_{i}=0$ or $\lambda_{i}, \lambda_{j} \neq 0$.

(4) If $X$ is not contained in a homogeneous hyperplane, meets any homogeneous hyperplane and $\operatorname{dim} X>1$, then, by Theorem $2, C h(X)$ is closed and, by Proposition 3, (SP) fails for $X$ on a pair of $T$-semiinvariant functions.

\section{Application to Binary FORMS}

Let char $k=0$. Consider the vector space $k[x, y]_{n}$ of binary forms of degree $n$, where $S L_{2}$ acts by the natural way and $k^{*}$ acts by homotheties.

$\mathrm{K}$. Baur proved the following theorem in [1].

Theorem [1, Th. 3.4]. Let $f \in k[x, y]_{n}$. Then the orbit $O_{f}=S L_{2} f$ satisfies $(S P)$ if and only if $f$ has a linear factor of multiplicity one.

We give the proof of this theorem here. Corollary 1 allows to simplify it (see Proposition 4 below).

The proof consists of some lemmas.

Lemma 2. Suppose that the orbit $O_{f}$ satisfies (SP). Then $f$ has a linear factor of multiplicity one.

Proof. Suppose that any linear factor of $f$ has the multiplicity no less then two. Note that this property holds for any $h(x, y)=h_{0} x^{n}+h_{1} x^{n-1} y+$ $\ldots+h_{n} y^{n} \in O_{f}$. Then (SP) fails for $O_{f}$ on the pair of linear functions $\alpha(h)=h_{0}, \beta(h)=h_{1}$. Indeed, if $h_{0}=0$ for some $h \in O_{f}$, then $h$ is divisible by $y$. This implies that $h$ is divisible by $y^{2}$ and $h_{1}=0$.

Lemma 3. The orbit $O_{f}$ satisfies (SP) if and only if the closure $\overline{k^{*} O_{f}}$ satisfies $(S P)$.

Proof. If $O_{f}$ has (SP), then $\overline{k^{*} O_{f}}$ has (SP) (as $O_{f} \subset \overline{k^{*} O_{f}}$ ).

Suppose that $O_{f}$ has not (SP), namely there exist homogeneous hyperplanes $H \neq H^{\prime}$ such that $H \cap O_{f} \subset H^{\prime}$. Then $H \cap t O_{f}=t\left(H \cap O_{f}\right) \subset H^{\prime}$ for any $t \in k^{*}$ and $H \cap k^{*} O_{f} \subset H^{\prime}$. By Lemma 4 below, it follows that $H \cap \overline{k^{*} O_{f}}=\overline{H \cap k^{*} O_{f}} \subset H^{\prime}$ and $\overline{k^{*} O_{f}}$ has not (SP).

Lemma 4. 4, Lemma 3(c)] Let $G: V$ be an irreducible representation of a connected algebraic group $G, H \subset V$ be a homogeneous hyperplane, and $X \subset V$ be a constructive $G$-invariant subset. Then $\overline{H \cap X}=H \cap \bar{X}$.

Proposition 4. The orbit $O_{x y^{n-1}}$ satisfies $(S P)$.

Proof. It is sufficient to prove that

$$
X=\overline{k^{*} S L_{2} x y^{n-1}}=\overline{G L_{2} x y^{n-1}}=\left\{(a x+b y)(c x+d y)^{n-1} \mid a, b, c, d \in k\right\}
$$

has the separation property (see Lemma 3$)$. For a point $h=(a x+b y)(c x+$ $d y)^{n-1} \in X$ we have

$$
\begin{aligned}
& h_{m}=a C_{n-1}^{m-1} c^{m-1} d^{n-m}+b C_{n-1}^{m} c^{m} d^{n-m-1}= \\
& =\frac{(n-1) ! c^{m-1} d^{n-m-1}(m a d+(n-m) b c)}{m !(n-m) !} .
\end{aligned}
$$

If the separation property does not hold, then $X$ is contained in a hypersurface of the form $h_{i}^{k}+h_{j} F=0$, where $F$ is a polynomial in the variables 
$h_{m}$ (see Corollary 1). Putting in this equation coordinates of points from $X$, we get a polynomial in the variables $a, b, c, d$ identically equal to zero. This implies that $h_{i}^{k}$ is divisible by $h_{j}$ (as a polynomial in $a, b, c, d$ ). It is easy to see that this can not be true for any $i, j, k$.

The following lemma combining with Lemma 3 completes the proof of the Theorem.

Lemma 5. If $f \in k[x, y]_{n}$ has a linear factor of multiplicity one, then $\overline{k^{*} O_{f}} \supseteq O_{x y^{n-1}}$.

Proof. We may assume that $f$ has the form $x\left(y+a_{2} x\right) \ldots\left(y+a_{n} x\right)$. Let $\gamma(t)$ be the diagonal matrix $\left(\begin{array}{cc}t & 0 \\ 0 & t^{-1}\end{array}\right) \in S L_{2}$. We have

$$
t^{n-2} \gamma(t) f=x\left(y+t^{2} a_{2} x\right) \ldots\left(y+t^{2} a_{n} x\right) \rightarrow x y^{n-1} \text { as } t \rightarrow 0 .
$$

Remark 6. Lemma 3 implies the similar result for the action $S L_{2}: \mathbb{P}\left(k[x, y]_{n}\right)$. Let $f \in \mathbb{P}\left(k[x, y]_{n}\right)$. Then the orbit $O_{f}=S L_{2} f$ satisfies (SP) if and only if $f$ has a linear factor of multiplicity one.

\section{The Closure of a toric orbit in a vector space}

Let $T$ be an algebraic torus and $\Lambda$ be the lattice of its characters. Consider a linear action $T: V$, where $t\left(x_{1}, \ldots, x_{n}\right)=\left(\chi_{1}(t) x_{1}, \ldots, \chi_{n}(t) x_{n}\right)$. Let $\Sigma$ be the semigroup in $\Lambda$ generated by the characters $\chi_{1}, \ldots, \chi_{n}$ and $K$ be the cone in $\Lambda \otimes_{\mathbb{Z}} \mathbb{Q}$ generated by $\Sigma$.

We are interested in the question if $X=\overline{T v}$ has the separation property. If the vector $v$ has a zero coordinate, then $X$ is contained in a homogeneous hyperplane and has not (SP). If any coordinate of $v$ is non-zero, then, up to the proportionality of the basis vectors, we may assume that $v=(1, \ldots, 1)$. It can also be assumed that the kernel of inefficiency of the action $T: V$ is finite (i.e., $\operatorname{dim} X=\operatorname{dim} T$, or the cone $K$ is bodily).

Lemma 6. [7. Lemma 4.1] Let $X=\overline{T(1, \ldots, 1)}$. The ideal $I(X)$ is generated (as a vector space) by all binomials of the form $x_{1}^{a_{1}} \cdot \ldots \cdot x_{n}^{a_{n}}-x_{1}^{b_{1}} \cdot \ldots \cdot x_{n}^{b_{n}}$, where $\sum a_{i} \chi_{i}=\sum b_{i} \chi_{i}, a_{i}, b_{i} \in \mathbb{Z}_{+}$.

Now we give an algorithm for a construction of a finite system of binomials defining $X$. Denote by $W$ the sublattice in $\mathbb{Z}^{n}$ defined by the system of equations $\sum c_{i} \chi_{i}=0$, where $c_{i} \in \mathbb{Z}$. To any point $c=\left(c_{1}, \ldots, c_{n}\right)$ of this sublattice we put in correspondence the binomial $x_{1}^{a_{1}} \cdot \ldots \cdot x_{n}^{a_{n}}-x_{1}^{b_{1}} \cdot \ldots \cdot x_{n}^{b_{n}}$, where $a_{i}=c_{i}, b_{i}=0$ if $c_{i}>0$, and $b_{i}=\left|c_{i}\right|, a_{i}=0$ otherwise. Such binomials vanish on $X$. Consider an octant $D_{I}=\left\{c \in \mathbb{Z}^{n} \mid c_{i} \geq 0\right.$ if $i \in I, c_{i} \leq 0$ if $i \notin I\}$. Then $W \cap D_{I}$ is a finitely generated semigroup. Let us construct a system of generators of $W \cap D_{I}$ (for example, if $d_{1}, \ldots, d_{k} \in W \cap D_{I}$ is a system of generators of the cone $\mathbb{Q}_{+}\left(W \cap D_{I}\right)$, then the set $\left\{\sum s_{i} d_{i} \mid 0 \leq s_{i} \leq 1\right\} \cap D_{I}$ generates $\left.W \cap D_{I}\right)$. Now we consider the union of such systems of generators of the semigroups $W \cap D_{I}$ over all octants and prove that the corresponding set of binomials generates the ideal $I(X)$. 
Indeed, the element $x^{I_{1}+J_{1}}-x^{I_{2}+J_{2}}$ belongs to the ideal generated by $x^{I_{1}}-x^{I_{2}}$ and $x^{J_{1}}-x^{J_{2}}$ :

$$
x^{I_{1}+J_{1}}-x^{I_{2}+J_{2}}=x^{J_{1}}\left(x^{I_{1}}-x^{I_{2}}\right)+x^{I_{2}}\left(x^{J_{1}}-x^{J_{2}}\right)
$$

(here $I_{1}, I_{2}, J_{1}, J_{2}$ are ordered sets of $n$ non-negative integers and $x^{J}=$ $x_{1}^{c_{1}} \ldots x_{n}^{c_{n}}$, where $\left.J=\left(c_{1}, \ldots, c_{n}\right), c_{i} \in \mathbb{Z}_{+}\right)$. Hence the binomials corresponding to the constructed system of generators of $W$ as a semigroup generate the ideal $I(X)$.

Theorem 3. The orbit closure $X=\overline{T(1, \ldots, 1)}$ satisfies $(S P)$ if and only if the following conditions hold:

(1) the cone $K$ is strictly convex;

(2) $\forall i \quad \mathbb{Q}+\chi_{i}$ is an edge of $K$;

(3) $\mathbb{Q}_{+} \chi_{i} \neq \mathbb{Q}_{+} \chi_{j}$ if $i \neq j$.

Remark 7. It is easy to see that these conditions are equivalent to the following:

(a) $\chi_{i} \notin<\chi_{1}, \ldots, \chi_{i-1}, \chi_{i+1}, \ldots, \chi_{n}>_{\mathbb{Q}_{+}}$for any $i$;

(b) $-\chi_{i} \notin<\chi_{1}, \ldots, \chi_{i-1}, \chi_{i+1}, \ldots, \chi_{n}>_{\mathbb{Q}_{+}}$for any $i$.

Proof. (1) If $K$ contains a line, then there exist $c_{1}, \ldots, c_{n} \in \mathbb{Z}_{+}$such that $c_{i} \neq 0$ for some $i$ and $\sum c_{i} \chi_{i}=0$. Therefore $X$ is contained in a hypersurface $x_{1}^{c_{1}} \cdot \ldots \cdot x_{n}^{c_{n}}=1$ and does not meet the hyperplane $x_{j}=0$, where $c_{j} \neq 0$. Consequently, $X$ has not (SP).

(2) If $\mathbb{Q}_{+} \chi_{i}$ is not an edge of $K$, then there exist $c_{1}, \ldots, c_{n} \in \mathbb{Z}_{+}$such that $c_{i} \chi_{i}=\sum_{j \neq i} c_{j} \chi_{j}, c_{i} \in \mathbb{N}$. If $\chi_{i}=0$, then $X$ does not meet the hyperplane $x_{i}=0$ and has not (SP). If $\chi_{i} \neq 0$, then there exists $c_{j} \neq 0, j \neq i$. This means that $x \in X, x_{j}=0$ implies $x_{i}=0$ and (SP) does not hold. The case $\mathbb{Q}_{+} \chi_{i}=\mathbb{Q}_{+} \chi_{j}$ can be proved by the same arguments.

(3) Now we prove the converse implication. Consider the case $\operatorname{dim} T=1$. The set $\left\{\chi_{1}, \ldots, \chi_{n}\right\}$ satisfies the conditions of the theorem only if $\operatorname{dim} V=$ 1. In this case $X=V$ if $\chi_{1} \neq 0$ and $X=\{1\}$ if $\chi_{1}=0$ and $X$ has (SP).

Now let $\operatorname{dim} X>1$. Note that $X$ is not contained in a hyperplane (otherwise there exist $i \neq j$ such that $\chi_{i}=\chi_{j}$ ) and meets any hyperplane (since $K$ is strictly convex and $\chi_{i} \neq 0$ for any $i$, it follows that $\left.0 \in X\right)$. Hence, by Theorem 2, $C h(X)$ is closed. If $X$ has not (SP), then, by Corollary $1, X$ is contained in the hypersurface $x_{i}^{l}+x_{j} F$ for some $i \neq j, F \in k[V], l \in \mathbb{N}$. By Lemma 6 , it follows that $x_{i}^{l}-x_{1}^{c_{1}} \ldots x_{n}^{c_{n}} \in I(X)$ for some $c_{1}, \ldots, c_{n} \in \mathbb{Z}_{+}$, $c_{j}>0$, i.e., $l \chi_{i}=\sum c_{m} \chi_{m}$. If $l \leq c_{i}$, then since $\chi_{j} \neq 0$, we have a contradiction with condition (1). Otherwise we have a contradiction with $\chi_{i} \notin<\chi_{1}, \ldots, \chi_{i-1}, \chi_{i+1}, \ldots, \chi_{n}>_{\mathbb{Q}_{+}}$.

Corollary 2. Let the orbit closure $X=\overline{T(1, \ldots, 1)}$ be defined by $p_{1}=0, \ldots, p_{m}=0$, where $p_{i}$ are binomials, and $S_{i}$ be the hypersurface defined by $p_{i}=0$. Then the following conditions are equivalent:

(1) $X$ does not satisfy (SP);

(2) there exists $i$ such that $S_{i}$ does not satisfy $(S P)$;

(3) there exists $i$ such that $p_{i}$ has either the form $x_{1}^{c_{1}} \ldots x_{n}^{c_{n}}-1$, where $c_{k} \geq 0$ and some $c_{j}>0$, or the form $x_{i}^{c_{i}}-x_{1}^{c_{1}} \ldots x_{i-1}^{c_{i-1}} x_{i+1}^{c_{i+1}} \ldots x_{n}^{c_{n}}$, where $c_{k} \geq 0, c_{i}>0$. 
Proof. The implications $(3) \Rightarrow(2)$ and $(2) \Rightarrow(1)$ are obvious.

Suppose that among $p_{i}$ there are no equations of such form. We shall prove that $X$ has (SP) applying Theorem 3.

(a) The cone $K$ is strictly convex and $\chi_{i} \neq 0$ for any $i$ since $0 \in X$.

(b) Suppose that $\mathbb{Q}_{+} \chi_{i}$ is not an edge of $K$ or $\mathbb{Q}_{+} \chi_{i}=\mathbb{Q}_{+} \chi_{j}$. This means that some equation of the form $x_{i}^{c_{i}}=x_{1}^{c_{1}} \ldots x_{i-1}^{c_{i-1}} x_{i+1}^{c_{i+1}} \ldots x_{n}^{c_{n}}$, where $c_{k} \geq 0, c_{i}>0$ and some $c_{j}>0, j \neq i$, vanishes on $X$. On the other hand, $\left(0, \ldots, x_{i}=1, \ldots, 0\right) \in X$ and we have a contradiction. By Theorem 3 , it follows that $X$ has (SP) and the implication $(1) \Rightarrow(3)$ is proved.

Example 7. Let $T=\left(k^{*}\right)^{3}$. Consider the 5-dimensional representation of $T$ with the characters $\chi_{1}=(1,0,0), \chi_{2}=(1,1,0), \chi_{3}=(0,1,2), \chi_{4}=(0,2,1)$, $\chi_{5}=(1,0,1)$. (On the picture below we draw the corresponding cone $K$.) Then $X=\overline{T(1,1,1,1,1)}$ can be defined by the equations

$$
\left\{\begin{array}{l}
x_{1}^{3} x_{3}=x_{2} x_{5}^{2}, \\
x_{1}^{3} x_{4}=x_{2}^{2} x_{5} \\
x_{2} x_{3}=x_{4} x_{5} .
\end{array}\right.
$$

The characters of this representation satisfy the conditions of Theorem 3 (and the equations do not satisfy the conditions of Corollary 2). Thus $X$ has the separation property.

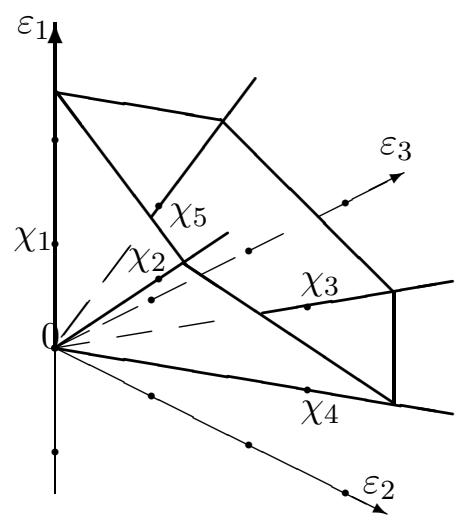

Remark 8. Note that an orbit of a torus has not (SP) $\left(x_{i} \neq 0\right.$ on $\left.T(1, \ldots, 1)\right)$.

Remark 9. We say that a variety $Y \subset V$ is binomial if $Y$ can be defined by a system of binomials. In particular, the closure of an orbit of a torus is binomial. Moreover, it is easy to see that a binomial variety is the closure of some toric orbit if and only if it is irreducible. The following example shows that the statement of Corollary 2 does not hold for an arbitrary binomial variety.

Example 8. Consider the binomial variety $Y \subset k^{4}$ defined by the equations

$$
\left\{\begin{array}{l}
x_{1} x_{2}=x_{3} x_{4}, \\
x_{1} x_{3}=x_{2} x_{4} \\
x_{1} x_{4}=x_{2} x_{3} .
\end{array}\right.
$$

Any hypersurface $x_{i} x_{j}=x_{l} x_{m}$ has (SP). It is easy to check that (SP) (and even (WSP)) for $Y$ fails on the functions $x_{1}-a x_{2}$ and $x_{1}-b x_{2}$, where 
$a, b \neq \pm 1, a \neq b$ (since $Y$ is an union of four coordinate axes and the lines $x_{1}= \pm x_{2}= \pm x_{3}= \pm x_{4}$ with an even number of minuses).

Theorem 4. The orbit closure $X=\overline{T(1, \ldots, 1)}$ satisfies (WSP) if and only if the following conditions hold:

(1) the cone $K$ is strictly convex;

(2) there are no more then one characters $\chi_{i}$ in the interior of any face of $K$ (in particular, $\mathbb{Q}_{+} \chi_{i} \neq \mathbb{Q}_{+} \chi_{j}$ for $i \neq j$ ).

Proof. (1) If $K$ contains a line, then there exist $c_{1}, \ldots, c_{n} \in \mathbb{Z}_{+}$such that $c_{j}, c_{k} \neq 0$ for some $j \neq k$ and $\sum c_{i} \chi_{i}=0$. Consequently, $X$ is contained in the hypersurface $x_{1}^{c_{1}} \cdot \ldots \cdot x_{n}^{c_{n}}=1$ and does not meet the hyperplanes $x_{j}=0$ and $x_{k}=0$. Therefore $X$ has not (WSP).

(2) Let condition (2) do not hold, i.e, there are two characters (let us assume that they are $\left.\chi_{1}, \chi_{2}\right)$ in the interior of the face of $K$ generated by $\left\{\chi_{i} \mid i \in I\right\}$. Then there exist $c_{i}, d_{i}>0$ such that $c_{1} \chi_{1}=\sum_{i \in I} c_{i} \chi_{i}$ and $d_{2} \chi_{2}=\sum_{i \in I} d_{i} \chi_{i}$. We have

$$
X \cap H_{x_{1}}=\cup_{i \in I} X \cap H_{x_{i}}=X \cap H_{x_{2}}
$$

and $X$ has not (WSP).

(3) Let us prove the converse implication. Consider the case $\operatorname{dim} T=1$. The cone $K$ satisfies the conditions of the theorem if and only if either $\operatorname{dim} V=1$ or $\operatorname{dim} V=2$ and $t\left(x_{1}, x_{1}\right)=\left(x_{1}, t^{m} x_{2}\right)$, where $m \neq 0$. In the first case $X$ has (SP) and it is easy to see that in the second case $X$ has (WSP).

Now let $\operatorname{dim} T \geq 2$. Assume the converse. Let the cone $K$ satisfy the conditions of Theorem 4 and the weak separation property fails for $X$ on linearly independent functions $\alpha=a_{1} x_{1}+\ldots+a_{n} x_{n}, \beta=b_{1} x_{1}+\ldots+b_{n} x_{n}$.

If $M$ is a cone generated by some edges of $K$ and $\delta$ is a linear function, then denote by $\delta_{M}$ the restriction of $\delta$ to the subspace in $V$ defined by the equations $x_{i}=0$, where $\chi_{i} \notin M$.

Remark 10. Let $L$ be a proper face of $K$ and $W \subset V$ be the subspace defined by the equations $x_{i}=0$, where $\chi_{i} \notin L$. Note that $X \cap W$ is the closure of the $T$-orbit of the point with the coordinates $x_{i}=1$ for $\chi_{i} \in L$ and $x_{i}=0$ for $\chi_{i} \notin L$. Since the characters of the representation $T: V$ satisfy the conditions of the theorem, it follows that the characters of the representation $T: W$ satisfy the same conditions. By inductions over $\operatorname{dim} V$, we may assume that $X \cap W \subset W$ has (WSP). Since $H_{\alpha_{L}} \cap X \cap W=H_{\beta_{L}} \cap X \cap W$, it follows that $\alpha_{L}$ and $\beta_{L}$ are linearly dependent.

Remark 11. If the linear functions $\tilde{\alpha}(x)=a_{1} x_{1}+\ldots+a_{i-1} x_{i-1}+a_{i+1} x_{i+1}+$ $\ldots+a_{n} x_{n}$ and $\tilde{\beta}(x)=b_{1} x_{1}+\ldots+b_{i-1} x_{i-1}+b_{i+1} x_{i+1}+\ldots+b_{n} x_{n}$ are proportional for some $i$, then for $x \in X$ we have $\alpha(x)=0 \Longleftrightarrow \beta(x)=$ $0 \Longleftrightarrow \tilde{\alpha}(x)=0$ and $x_{i}=0$. There exists a one-parameter subgroup $\gamma: k^{*} \rightarrow T$ such that $\left\langle\gamma, \chi_{r}\right\rangle \neq\left\langle\gamma, \chi_{j}\right\rangle$ for $r \neq j$. Hence there exists a non-zero root of the equation $a_{1} t^{\left\langle\gamma, \chi_{1}\right\rangle}+\ldots+a_{n} t^{\left\langle\gamma, \chi_{n}\right\rangle}=0$. Thus we have a contradiction.

The first case. Suppose that $\chi_{i} \neq 0$ for any $i$. 
Step 1. Suppose that $\alpha(x)=a_{i} x_{i}+a_{j} x_{j}$ and $\beta(x)=b_{i} x_{i}+b_{j} x_{j}$. Note that $a_{i} x_{i}+a_{j} x_{j}=0$ and $b_{i} x_{i}+b_{j} x_{j}=0$ if and only if $x_{i}=0, x_{j}=0$. Since $\chi_{i} \neq \chi_{j}$, it follows that there exists $x \in T(1, \ldots, 1)$ such that $\alpha(x)=0$ and we have a contradiction.

Step 2. Suppose that $\alpha(x)=a_{i} x_{i}+a_{j} x_{j}+a_{m} x_{m}$ and $\beta(x)=b_{i} x_{i}+b_{j} x_{j}+$ $b_{m} x_{m}$, where $\chi_{m}$ belongs to the interior of $K$.

1. If $a_{i}=b_{i}=0$ or $a_{j}=b_{j}=0$, then we have a contradiction (see Step 1).

2. If $a_{i}=0, b_{i} \neq 0$, then there exists a one-parameter subgroup $\gamma: k^{*} \rightarrow T$ such that $\gamma(s) \alpha \rightarrow \alpha^{\prime}, \gamma(s) \beta \rightarrow \beta^{\prime}$, where the supports of $\alpha^{\prime}$ and $\beta^{\prime}$ do not intersect. Since $C h w(X)$ is closed, it follows that $\left(\left\langle\alpha^{\prime}\right\rangle,\left\langle\beta^{\prime}\right\rangle\right) \in C h w(X)$ and $\overline{T\left(\left\langle\alpha^{\prime}\right\rangle,\left\langle\beta^{\prime}\right\rangle\right)} \cap D=\varnothing$. There exists a $T$-stable point $\left(\left\langle x_{l}\right\rangle,\left\langle x_{r}\right\rangle\right) \in$ $\overline{T\left(\left\langle\alpha^{\prime}\right\rangle,\left\langle\beta^{\prime}\right\rangle\right)}$. Consequently, $X$ is contained in a hypersurface of the form $x_{l}^{c}+x_{r}^{d}+x_{l} x_{r} F$ for some $l \neq r, c, d \in \mathbb{N}, F \in k[X]$. Lemma 5 implies that the binomials $x_{l}^{c}-x_{1}^{c_{1}} \ldots x_{n}^{c_{n}}$ and $x_{r}^{d}-x_{1}^{d_{1}} \ldots x_{n}^{d_{n}}$ belong to $I(X)$ for some $c_{l}, d_{l}$ such that $c_{r}, d_{l}>0$ and $c \chi_{l}=\sum c_{i} \chi_{i}, d \chi_{r}=\sum d_{i} \chi_{i}$. If $c \leq c_{l}$ or $d \leq d_{r}$, then this contradicts condition (1). Otherwise $\chi_{l}$ and $\chi_{r}$ belong to the interior of the same face of $K$ and we also have a contradiction. The cases when $a_{i} \neq 0, b_{i}=0$ or $a_{j}=0, b_{j} \neq 0$ or $a_{j} \neq 0, b_{j}=0$ can be considered by the same way.

3. Now let $a_{i}, b_{i}, a_{j}, b_{j} \neq 0$. It can be assumed that $a_{i}=b_{i}=1$. There exists a one-parameter subgroup $\gamma: k^{*} \rightarrow T$ such that $\left\langle\gamma, \chi_{i}\right\rangle=n_{1}>\left\langle\gamma, \chi_{m}\right\rangle=$ $\left.n_{2}\right\rangle\left\langle\gamma, \chi_{j}\right\rangle=0$. The equations

$$
s^{n_{1}}+a_{m} s^{n_{2}}+a_{j}=0
$$

and

$$
s^{n_{1}}+b_{m} s^{n_{2}}+b_{j}=0 \quad(* *)
$$

have the same roots up to multiplicities. If all roots of one equation have the multiplicity one, then all roots of another equation have the multiplicity one and the equations are proportional. A multiple root $s_{0}$ of $(*)$ is a root of its derivative and satisfies the equation

$$
s_{0}^{n_{1}-n_{2}}=-\frac{a_{m} n_{2}}{n_{1}} .
$$

(If $n_{1}$ is divisible by char $k$, then either $a_{m}=b_{m}=0$ and this is Step 1 or $n_{2}$ is divisible by char $k$. In the second case we replace $n_{1}$ by $\frac{n_{1}}{\text { char } k}, n_{2}$ by $\frac{n_{2}}{\text { chark } k}$, and $a_{j}, a_{m}, b_{j}, b_{m}$ by the roots of degree char $k$ and obtain similar equations.) Putting obtained formulæ in $(*)$, we get

$$
s_{0}^{n_{2}}\left(-\frac{a_{m} n_{2}}{n_{1}}\right)+a_{m} s_{0}^{n_{2}}+a_{j}=0
$$

This implies

$$
s_{0}^{n_{2}}=-\frac{a_{j} n_{1}}{a_{m}\left(n_{1}-n_{2}\right)}
$$

(if $a_{m}=0$ or $n_{1}-n_{2}$ is divisible by char $k$, then $n_{1}$ is divisible by char $k$ and we repeat the previous arguments). Also, we have

$$
s_{0}^{n_{1}}=\frac{a_{j} n_{2}}{n_{1}-n_{2}} .
$$

Note that $s_{0}$ is a root of $(* *)$. Putting obtained formulæ in $(* *)$, we get

$$
a_{m} a_{j} n_{2}-b_{m} a_{j} n_{1}+b_{j} a_{m}\left(n_{1}-n_{2}\right)=0 .
$$


Since $(* *)$ also has a multiple root, then, by the same arguments, we have the symmetric equation

$$
b_{m} b_{j} n_{2}-a_{m} b_{j} n_{1}+a_{j} b_{m}\left(n_{1}-n_{2}\right)=0 .
$$

Summing the obtained equations, we get $\left(a_{j}-b_{j}\right)\left(a_{m}-b_{m}\right)=0$. Thus we have a contradiction with Remark 11.

Step 3. Consider the case of arbitrary $\alpha, \beta$. In the case $\operatorname{dim} T=2$ we have a contradiction with Step 2.

Now let $\operatorname{dim} T>2$. We say that $\tilde{K}$ is a subcone of $K$ if $\tilde{K}$ is generated by a finite number of vectors from $K$. Note that the interior of a subcone is contained in the interior of one of the faces of $K$. Consider the subcone $K^{\prime}$ of $K$ generated by the characters $\chi_{i}$ such that $a_{i} \neq 0$ or $b_{i} \neq 0$. Since $\alpha_{K^{\prime}}$ and $\beta_{K^{\prime}}$ are not proportional, it follows that there exists a face $L_{1}$ of codimension 1 in $K^{\prime}$ such that $\alpha_{L_{1}}$ and $\beta_{L_{1}}$ are not proportional. If $\chi_{m}$ is contained in the interior of $L_{1}$, then $\chi_{m}$ is contained in the interior of $K$. Indeed, assume the converse. Let $\chi_{i}$ be an interior point of a proper face of $K$ and $\chi_{i}$ belong to the interior of $L_{1}$. Then $L_{1}$ is contained in this face of $K$. By Remark 10, the linear functions $\alpha_{L_{1}}$ and $\beta_{L_{1}}$ are proportional and we have a contradiction. For the same reason, there exists a face $L_{2}$ of codimension one in $L_{1}$ such that $\alpha_{L_{2}}$ and $\beta_{L_{2}}$ are not proportional. Thus we can find a 2 -dimensional face $L_{r}$ with this property. There exists a oneparameter subgroup $\gamma: k^{*} \rightarrow T$ such that $\gamma(s) \alpha \rightarrow \alpha_{L_{r}}, \gamma(s) \beta \rightarrow \beta_{L_{r}}$ as $s \rightarrow 0$. Since $C h w(X)$ is closed, it follows that $\left(\left\langle\alpha_{L_{r}}\right\rangle,\left\langle\beta_{L_{r}}\right\rangle\right) \in C h w(X)$ and we can apply Step 2 .

The second case. Suppose that $\chi_{i}=0$ for some $i$ (we may assume that $i=1$ ). If $a_{1}=b_{1}=0$, then consider the image $X^{\prime} \subset W$ of $X$ under the projection along the first basis vector (here $W$ is a subspace defined by the equation $\left.x_{1}=0\right)$. The characters of the representation $T: W$ are nonzero and satisfy the conditions of the theorem. The first case implies that $X^{\prime} \subset W$ has (WSP). But (WSP) fails for $X^{\prime} \subset W$ on the restriction on $W$ of the functions $\alpha, \beta$. This is a contradiction.

Let $a_{1}$ or $b_{1}$ be not equal to zero. By Remark 10, it follows that the vector $\left(a_{i}, b_{i}\right)$ is proportional to the vector $\left(a_{1}, b_{1}\right)$ for $i \neq m$. Thus the linear functions $\alpha^{\prime}=a_{1} x_{1}+\ldots+a_{m-1} x_{m-1}+a_{m+1} x_{m+1}+\ldots+a_{n} x_{n}$ and $\beta^{\prime}=b_{1} x_{1}+\ldots+b_{m-1} x_{m-1}+b_{m+1} x_{m+1}+\ldots+b_{n} x_{n}$ are proportional. This contradicts to Remark 11.

Theorem 5. Suppose that the orbit closure $X=\overline{T(1, \ldots, 1)} \subset V$ is a cone; then $X$ satisfies (SSP) if and only if $X=V$ (i.e., the weights $\chi_{1}, \ldots, \chi_{n}$ are linearly independent).

Proof. Let $X \neq V$ and $x_{1}^{a_{1}} \ldots x_{n}^{a_{n}}-x_{1}^{b_{1}} \ldots x_{n}^{b_{n}} \in I(X)$, where there exists $i$ with $a_{i} \neq b_{i}$. We may assume that $a_{i}=0$ or $b_{i}=0$ for any $i$. Let $a_{1}>0$. Since $X$ is a cone, it follows that there exists $b_{i}>0$ and

$$
X \cap H_{x_{1}}=\cup_{i: b_{i}>0}\left(X \cap V_{i}\right),
$$

where $V_{i}=H_{x_{1}} \cap H_{x_{i}}$. This implies that there exists $i$ such that $\operatorname{dim} X \cap V_{i}=\operatorname{dim} X \cap H_{x_{1}}$ and $X \cap V_{i}$ has the codimension $\leq 1$ in $X$. 
Remark 12. The proof of Theorem 5 is true for any cone which is contained in a binomial hypersurface, in particular, for binomial cones.

Question. Let $X$ be a closed irreducible $T$-invariant subvariety in a $T$ module $V$ such that $X$ has not (WSP) (resp. (SSP)). Is it true that (WSP) (resp. (SSP)) for $X$ fails on a pair of $T$-semiinvariant linear functions?

\section{The Closure of A TORIC ORBIT IN A PROJECTIVE SPACE}

Let $T: \mathbb{P}(V), t\left(x_{1}: \ldots: x_{n}\right)=\left(\chi_{1}(t) x_{1}: \ldots: \chi_{n}(t) x_{n}\right)$. We are interested in the question if $Y=\overline{T w}(w \in \mathbb{P}(V))$ has the separation properties. As in the previous section we may assume that $w=(1: \ldots: 1)$. It also can be assumed that the kernel of inefficiency of the action $T: \mathbb{P}(V)$ is finite, i.e., $\operatorname{dim} X=\operatorname{dim} T$.

Theorem 3'. The orbit closure $X=\overline{T(1: \ldots: 1)}$ satisfies (SP) if and only if the following conditions hold:

(1) the point $\chi_{i}$ is a vertex of the convex hull conv $\left\{\chi_{1}, \ldots, \chi_{n}\right\}$ for any $i$;

(2) $\chi_{i} \neq \chi_{j}$ for $i \neq j$.

Proof. Consider the action $T \times k^{*}: V$, where

$$
(t, s)\left(x_{1}, \ldots, x_{n}\right)=\left(s \chi_{1}(t) x_{1}, \ldots, s \chi_{n}(t) x_{n}\right) .
$$

The weights of this representation are $\chi_{i}^{\prime}=\chi_{i}+\lambda$, where $\lambda(t, s)=s$. Then

$$
T(1: \ldots: 1)=\mathbb{P}\left(T \times k^{*}(1, \ldots, 1)\right),
$$

and $X=\overline{T(1: \ldots: 1)}=\overline{\mathbb{P}\left(T \times k^{*}(1, \ldots, 1)\right)}$. We shall apply Theorem 4 to $\overline{T \times k^{*}(1, \ldots, 1)}$.

The cone $K$ is strictly convex since $c_{i} \geq 0$ and $\sum c_{i} \chi_{i}^{\prime}=0$ implies $c_{i}=0$ for any $i$. Further, $\chi_{i}^{\prime}$ is an edge of $K$ (i.e., $\left.\chi_{i}^{\prime} \notin<\chi_{1}^{\prime}, \ldots, \chi_{i-1}^{\prime}, \chi_{i+1}^{\prime}, \ldots, \chi_{n}^{\prime}>_{\mathbb{Q}_{+}}\right)$if and only if $\chi_{i}$ is not contained in the convex hull of the set $\left\{\chi_{1}, \ldots, \chi_{i-1}, \chi_{i+1}, \ldots, \chi_{n}\right\}$. Finally, $\mathbb{Q}_{+} \chi_{i}^{\prime} \neq \mathbb{Q}_{+} \chi_{j}^{\prime}$ is equivalent to $\chi_{i} \neq \chi_{j}$.

Remark 13. If any hyperplane section of $X \subset \mathbb{P}(V)$ is reduced (i.e., it is a sum of prime divisors), then $X$ has (SP) (see 4, Lemma 2]). If $X$ is the orbit of a highest vector in an irreducible representation of a reductive group, then this condition is equivalent to (SP) (see 4, Prop. 5]). This is not true for an orbit closure of a torus. Consider the action of the torus $T=\left(k^{*}\right)^{2}: \mathbb{P}\left(k^{4}\right),\left(t_{1}, t_{2}\right)\left(x_{0}: x_{1}: x_{2}: x_{3}\right)=\left(t_{1} t_{2}^{2} x_{0}, t_{1} t_{2} x_{1}, t_{1}^{3} x_{2}, t_{2}^{2} x_{3}\right)$. The orbit closure of the point $(1: 1: 1: 1)$ is the hypersurface defined by the equation $x_{0} x_{1}^{2}=x_{2} x_{3}^{2}$. It has (SP) and its intersection with $H_{x_{0}}$ is not reduced.

Theorem 4'. The orbit closure $X=\overline{T(1: \ldots: 1)}$ satisfies (WSP) if and only if there are no more then one $\chi_{j}$ in the interior of any face of the convex hull conv $\left\{\chi_{1}, \ldots, \chi_{n}\right\}$ (in particular, $\chi_{i} \neq \chi_{j}$ for $i \neq j$ ).

Proof. The proof is similar to the proof of Theorem 3'.

Theorem 5'. The orbit closure $X=\overline{T(1: \ldots: 1)} \subset \mathbb{P}(V)$ satisfies $(S S P)$ if and only if $X=\mathbb{P}(V)$ (i.e., the weights $\chi_{1}, \ldots, \chi_{n}$ are affinely independent).

Proof. The proof follows from Theorem 5. 


\section{REFERENCES}

[1] K. Baur, Two Contributions to the Representation Theory of Algebraic Groups, Doctoral Thesis, Basel, 2002.

[2] W. Fulton, Introduction to Toric Varieties. Annals of Math. Studies 131, Princeton University Press, 1993.

[3] J. Humphreys, Linear algebraic groups. Graduate Texts in Mathematics 21, New York - Heidelberg - Berlin: Springer-Verlag, 1975.

[4] H. Kraft, N.R. Wallach, On the separation property of orbits in representation spaces, Journal of Algebra 258 (2002), 228-254.

[5] A. Premet, Support varieties of non-restricted modules over Lie algebras of reductive groups, J. London Math. Soc. 55:2 (1997), 236-250.

[6] I. R. Shafarevich, Basic algebraic geometry. Berlin: Springer-Verlag, 1994.

[7] B. Sturmfels, Gröbner Bases and Convex Polytopes. University Lecture Series, Volume 8, 1995.

Department of Higher Algebra, Faculty of Mechanics and Mathematics, Moscow State University, 119992 Moscow, Russia

E-mail address: chuvashova@yandex.ru 